04,06

\title{
Сравнение характеристик тонких пленок PZT на подложках из сапфира и кремния
}

\author{
() Л.А. Делимова ${ }^{1}$, Н.В. Зайцева ${ }^{1}$, В.В. Ратников ${ }^{1}$, В.С. Юферев ${ }^{1}$, Д.С. Серегин ${ }^{2}$, \\ К.А. Воротилов ${ }^{2}$, А.С. Сигов ${ }^{2}$ \\ ${ }^{1}$ Физико-технический институт им. А.Ф. Иофрфе, \\ Санкт-Петербург, Россия \\ ${ }^{2}$ МИРЭА - Российский технологический университет, \\ Москва, Россия \\ E-mail: ladel@mail.ioffe.ru
}

Поступила в Редакцию 13 марта 2021 г.

В окончательной редакции 14 марта 2021 г.

Принята к публикации 14 марта 2021 г.

Свойства сегнетоэлектрических золь-гель-пленок PZT, осажденных на подложку из тонкого слоя кремния на сапфире „Si-on-Sapphire“ (SOS) сравниваются с пленками PZT, сформированными на подложке из кремния. Исследованы кристаллическая структура методом рентгеновской дифракции, асимметрия петель гистерезиса, поляризационные зависимости переходного тока, короткозамкнутого фототока и фото-ЭДС холостого хода, а также изгиб подложек. Пленки PZT на SOS текстурированы в единственном направлении (111) и демонстрируют симметричные петли гистерезиса с высоким значением остаточной поляризации. Пленки PZT на Si текстурированы в основном (111) и более слабом (100) направлениях, показывают меньшие значения поляризации и проявляют асимметрию петель гистерезиса, что отражается на величине переходного тока и фототока. Показано, что сапфировая подложка имеет выпуклый изгиб, вызывающий в плоскости пленки напряжение сжатия, ослабляющее влияние рассогласования параметров решеток PZT и Pt. Напротив, подложка Si имеет вогнутый изгиб, вызывающий растяжение пленки. Сделаны оценки деформаций и механических напряжений внутри пленок. Для PZT на Si получена оценка градиента деформации вдоль оси (111), которая позволяет связать асимметрию петель гистерезиса с флексоэлектрической поляризацией, и найдена величина флексоэлектрического коэффициента для золь-гель-РZT, равная $0.0154 \mu \mathrm{C} / \mathrm{cm}$. Полученные результаты показывают, что использование сапфировой подложки обеспечивает лучшее качество тонких пленок PZT.

Ключевые слова: сегнетоэлектрические пленки, PZT, непереключаемая поляризация, петли гистерезиса, вольтамперные характеристики, фототок, механические деформации и напряжения.

DOI: 10.21883/FTT.2021.08.51157.052

\section{1. Введение}

Тонкие пленки цирконата титаната свинца (PZT) представляют большой интерес благодаря высоким значениям спонтанной поляризации и пьезоэлектрических коэффициентов при относительно низкой температуре кристаллизации. Это делает материал PZT чрезвычайно привлекательным для создания различных видов интегрированных сегнетоэлектрических устройств. Например, сегнетоэлектрическая (СЭ) энергонезависимая память FeRAM (Ferroelectric Random Access Memory) на основе PZT нашла применение на рынке, оказавшись востребованной в устройствах памяти с низким энергопотреблением/низким напряжением $[1,2]$. Микроэлектромеханические системы (MEMS) на основе PZT представляют особый интерес из-за возможности достигать относительно больших перемещений исполнительных элементов при небольших управляющих напряжениях [3-5]. Для изготовления таких устройств обычно используются стандартные монокристаллические или эпитаксиальные пластины кремния $[3,6]$. Пластины крем- ния на изоляторе „Silicon-on-Insulator“ (SOI) широко используются в технологии производства полупроводников для снижения энергопотребления CMOS- и MEMSустройств $[7,8]$. Структуры $\mathrm{Si}-\mathrm{SiO}_{2}-\mathrm{Si}$, полученные путем сращивания пластин кремния и оксида кремния, являются наиболее распространенным видом подложек SOI [9], гетероэпитаксиальный Si, выращенный на сапфировой подложке, „Silicon-on-Sapphire“ (SOS) встречается реже, хотя SOS является исторически первой из SOI технологий $[10,11]$.

Есть много сообщений об интеграции пленок PZT с технологией SOI, преимущественно, при производстве устройств MEMS, см., например, [12-14], но мало известно о пленках PZT, выращенных на пластинах SOS $[3,15,16]$. Есть информация, что PZT на SOS использовались для создания устройств FeRAM [15] и радиочастотных микроэлектромеханических систем RF MEMS [3,16]. Некоторые исследования посвящены изучению свойств пленок PZT, нанесенных непосредственно на платинированные сапфировые подложки [17-19]. Недавно в работе [16] сообщалось, что 
пленки PZT на SOS демонстрируют несколько лучшие сегнетоэлектрические свойства, чем на объемном кремнии. Авторы предполагают, что причина кроется в более высоком коэффициенте теплового расширения сапфира, который приводит к большому сжатию пленок PZT, тогда как на массивном $\mathrm{Si}$ пленки испытывают напряжение растяжения. Однако физические свойства таких структур были изучены недостаточно, без исследования физических процессов в пленках PZT на SOS.

Целью настоящей работы является комплексное исследование электрофизических свойств сегнетоэлектрических пленок PZT, нанесенных на подложку SOS, в сравнении с PZT на подложке Si. Оно включает исследования кристаллической структуры методами рентгеновской дифракции (XRD), асимметрии петель гистерезиса, поляризационных зависимостей переходного тока, короткозамкнутых фототоков и фото-ЭДС холостого хода, а также изгиба подложки и оценки деформаций и механических напряжений внутри пленок.

\section{2. Образцы}

Тонкие пленки $\mathrm{Pb}\left(\mathrm{Zr}_{0.48} \mathrm{Ti}_{0.52}\right) \mathrm{O}_{3}$ с избытком $\mathrm{Pb}$ $15 \mathrm{wt} \%$ в пленкообразующем растворе формировались на $R$-срезе $\mathrm{Al}_{2} \mathrm{O}_{3}(510 \mu \mathrm{m}) / \mathrm{Si}(600 \mathrm{~nm}) / \mathrm{SiO}_{2}(300 \mathrm{~nm}) /$ $\mathrm{TiO}_{2}(10 \mathrm{~nm}) / \mathrm{Pt}(150 \mathrm{~nm})$ подложки методом осаждения из химического раствора, подробности которого приведены в ссылке [20]. Температура отжига пленки составляла $650^{\circ} \mathrm{C}$. Полупрозрачные верхние платиновые электроды толщиной $10 \mathrm{~nm}$ и площадью $10^{-3} \mathrm{~cm}^{2}$ были сформированы методом магнетронного распыления на постоянном токе. Для сравнения пленки PZT формировались из того же раствора в тех же условиях на подложке $\mathrm{Si}(690 \mu \mathrm{m}) / \mathrm{SiO}_{2}(300 \mathrm{~nm}) / \mathrm{TiO}_{2}(10 \mathrm{~nm}) /$ $\operatorname{Pt}(150 \mathrm{~nm})$ производства Inostec, Корея. Толщина пленок, найденная с помощью эллипсометра Sentech-850, составила 150 и $210 \mathrm{~nm}$ для PZT на SOS и $\mathrm{Si}$ соответственно.

Структура пленок исследовалась на дифрактометpe ДРОН-3 (режим сканирования $\Theta-2 \Theta$, излучение $\left.\mathrm{Cu} K_{\alpha 1}\right)$. Петли гистерезиса измеряли методом Сойера-Тауэра с разверткой синусоидального напряжения в диапазоне $\pm 6 \mathrm{~V}$ и частотой сигнала $64 \mathrm{~Hz}$. Поляризационные зависимости переходного тока и фототока измеряли с помощью источника напряжения и пикоамперметра Keithley 6487.

\section{3. Экспериментальные результаты и их обсуждение}

\subsection{XRD-исследования}

На рис. 1 приведены пики рентгеновской дифракции, измеренные в пленках PZT, сформированных на подложках SOS и $\mathrm{Si}$. Видно, что пленка PZT на $\mathrm{Si}$ текстурирована в основном направлении (111) и в более

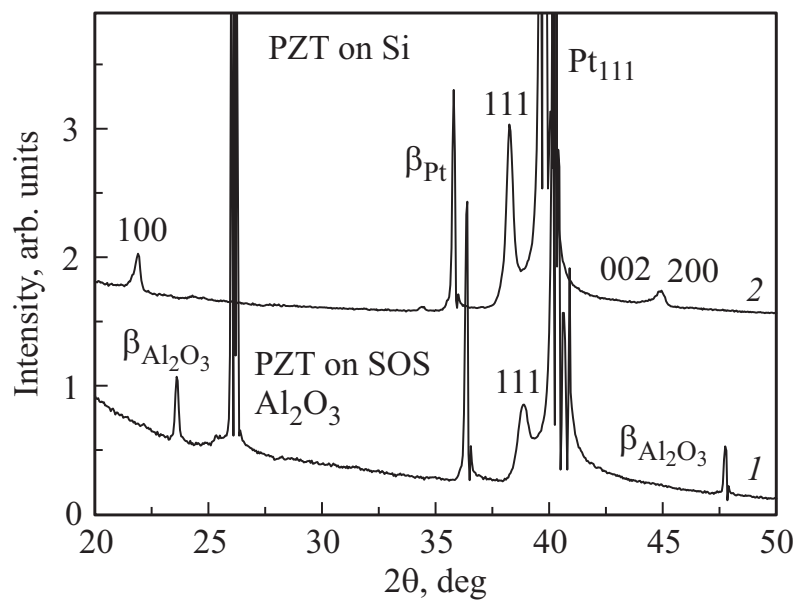

Рис. 1. Дифрактограммы PZT-пленок, осажденных на подложки $\operatorname{SOS}(1)$ и $\mathrm{Si}(2)$.

слабых направлениях (100) и (002). Из полуширин пиков рентгеновской дифракции (200) и (002) были определены усредненные по объему пленки параметры тетрагональной решетки $a=0.4042 \mathrm{~nm}$ и $c=0.4104 \mathrm{~nm}$ соответственно. Заметим, что для ненапряженной решетки PZT того же состава значение параметра решетки меньше вдоль оси $(100), a^{*}=0.4025 \mathrm{~nm}$, и больше вдоль оси $(001), c^{*}=0.4140 \mathrm{~nm}$, поэтому можно считать, что пленка была растянута в плоскости $\{110\}$ и сжата в направлении (001). Пленка PZT на SOS имеет единственную текстуру в направлении (111). Постоянная решетки платины определялась из полуширины дифракционного пика $\mathrm{Pt}_{222}$ и оказалась равной $0.39229 \pm 0.00006 \mathrm{~nm}$ для SOS подложки и $0.3918 \pm 0.0005 \mathrm{~nm}$ для Pt на Si. Первое значение совпадает с табличными данными $(0.3923 \mathrm{~nm})$, в то время как второе оказывается заметно меньше, что указывает на деформацию сжатия. Значения деформаций $\delta a / a$, определенные по формуле $\delta a / a=B /\left(4 \operatorname{tg} \theta_{222}\right)$, где $B$ - полуширина пика $\mathrm{Pt}_{222}$, составили 0.0006 и 0.0014 для Pt на SOS и на Si соответственно. Размеры зерен $\mathrm{Pt}$, оцененные по полуширине рефлекса $\mathrm{Pt}_{111}$, составили 100 и $55 \mathrm{~nm}$ для $\mathrm{Pt}$ на $\mathrm{SOS}$ и на $\mathrm{Si}$.

\section{2. Петли гистерезиса}

Для изучения природы асимметрии петель гистерезиса использовался метод Сойера-Тауэра, дополненный процедурой деполяризации путем подачи затухающего синусоидального напряжения на структуру $\mathrm{Pt} / \mathrm{PZT} / \mathrm{Pt}$, включенную последовательно с эталонным конденсатором емкостью $220 \mathrm{nF}$. При этом, положительным считалось направление от нижнего электрода к верхнему, который заземлен, а поляризация регистрировалась по величине заряда, наведенного на электроде эталонного конденсатора при переключении СЭ-доменов под действием напряжения. Амплитуда синусоидального напряжения уменьшалась от $6 \mathrm{~V}$ до нуля в течение 20 периодов длительностью $0.3 \mathrm{~s}$. 

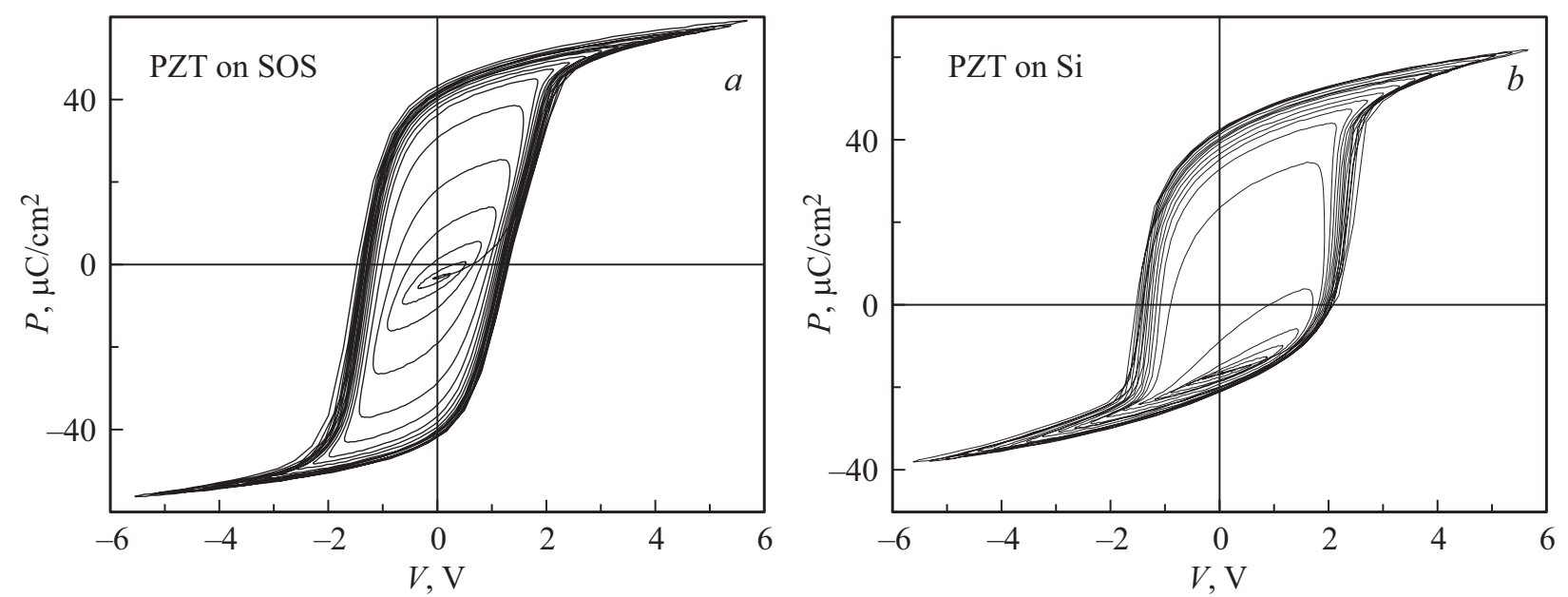

Рис. 2. Семейство гистерезисных петель, измеренное методом Сойера-Тауэра в пленках PZT, осажденных на подложки SOS $(a)$ и $\mathrm{Si}(b)$.

Семейство деполяризующих петель пленки PZT на SOS показано на рис. $2, a$. Видно, что петли практически симметричны, показывают высокие значения остаточной поляризации $P_{R}^{+}=+42 \mu \mathrm{C} / \mathrm{cm}^{2}, P_{R}^{-}=-40 \mu \mathrm{C} / \mathrm{cm}^{2}$ с коэрцитивной силой $\sim 100 \mathrm{kV} / \mathrm{cm}$. В деполяризованном состоянии, когда внешнее напряжение становится равным нулю, величина остаточной поляризации $P_{\text {dep }} \sim 3.2 \mu \mathrm{C} / \mathrm{cm}^{2}$; для разных пленок $P_{\text {dep изменялась }}$ в пределах $-(2-4) \mu \mathrm{C} / \mathrm{cm}^{2}$.

В противоположность этому, петли гистерезиса PZT на $\mathrm{Si}$ сильно асимметричны (рис. 2, $b$ ): коэрцитивная сила составляет $-70 \mathrm{kV} / \mathrm{cm}$ и $+100 \mathrm{kV} / \mathrm{cm}$, остаточная поляризация $P_{R}^{+}=+42 \mu \mathrm{C} / \mathrm{cm}^{2}, \quad P_{R}^{-}=-21 \mu \mathrm{C} / \mathrm{cm}^{2}$, a поляризация в деполяризованном состоянии $P_{\text {dep }}=-16 \mu \mathrm{C} / \mathrm{cm}^{2}$; для разных структур она изменялась в пределах $P_{\text {dep }} \sim-(10-16) \mu \mathrm{C} / \mathrm{cm}^{2}$. Можно видеть, что в обоих случаях остаточная положительная поляризация $P_{R}^{+}$больше отрицательной $P_{R}^{-}$по абсолютной величине. Однако если для PZT на SOS эта разница незначительна, то для PZT на $\mathrm{Si}$ она оказывается сопоставимой с $P_{R}^{-}$. Превышение $P_{R}^{+}$над $P_{R}^{-}$предполагает, что в СЭ-пленке имеется непереключаемая положительная поляризация (или какой-то иной заряд на интерфейсе между нижним электродом и PZT), отражением чего является поляризация $P_{\mathrm{dep}}$ в деполяризованном состоянии. В качестве оценки величины непереключаемой поляризации можно взять $P_{n o n-s w}=\left(P_{R}^{+}+P_{R}^{-}\right) / 2$. Для PZT на SOS, как уже отмечалось, поляризация $P_{n o n-r w}$ оказывается незначительной $\left(+1 \mu \mathrm{C} / \mathrm{cm}^{2}\right)$, в то время как для PZT на $\mathrm{Si}$ достаточно велика $\left(+10.5 \mu \mathrm{C} / \mathrm{cm}^{2}\right)$. Появление отрицательной остаточной поляризации $P_{\text {dep }}$ при наличии положительной непереключаемой поляризации $P_{\mathrm{dep}}$, на первый взгляд, кажется удивительным, но оно вполне объяснимо. Как следует из рис. $2, b$, в случае PZT на $\mathrm{Si}$ максимальная поляризация всех элементарных доменов, способных к переключению, $P_{s w}^{\max }=P_{R}^{+}-P_{n o n-s w}=31.5 \mu \mathrm{C} / \mathrm{cm}^{2}$.
В деполяризованном состоянии одна часть этих доменов поляризована отрицательно, а другая - положительно, с поляризациями $P_{s w}^{-}$и $P_{s w}^{+}$соответственно. Так как $P_{s w}^{\max }=P_{s w}^{+}+\left|P_{s w}^{-}\right| \quad$ и $\quad P_{\mathrm{dep}}=P_{n o n-s w}+P_{s w}^{+}-\left|P_{s w}^{-}\right|$, получаем $P_{s w}^{-}=-29$ и $P_{s w}^{+}=2.5 \mu \mathrm{C} / \mathrm{cm}^{2}$. Аналогичные рассуждения, примененные к PZT на SOS, дают -22.6 и $18.4 \mu \mathrm{C} / \mathrm{cm}^{2}$ соответственно. Наличие большой непереключаемой поляризации в $\mathrm{PZT}$ на $\mathrm{Si}$, вопервых, вызывает значительное (на 25\%) уменьшение величины переключаемого поляризационного заряда (а, следовательно, и тока переключения) по сравнению с PZT на SOS, а во-вторых, является возможной причиной возникновения весьма значительного отрицательного встроенного поля, которое приводит к асимметрии гистерезисной петли по оси напряжений.

\section{3. Измерение переходного тока}

При измерении вольтамперных зависимостей РZTструктур смещение прикладывается как последовательность ступенек амплитудой $0.1 \mathrm{~V}$ и длительностью $0.2 \mathrm{~s}$, a ток регистрируется в конце ступени. Амплитуда напряжения изменялась от 0 до $5 \mathrm{~V}$, затем обратно к 0 . В схеме измерения время переключения доменов составляет $\sim 2 \mathrm{~ms}$, и поэтому к моменту регистрации тока (через $200 \mathrm{~ms}$ после приложения смещения) вызванный переключением доменов ток уже протек через внешнюю цепь. В результате в описываемом эксперименте измеряется ток, связанный с движением подвижных носителей заряда и изменением электрического поля во времени.

Для обоих направлений смещения измерения выполнялись при разных предварительных поляризациях. Перед каждым измерением пленка деполяризовалась, затем поляризовалась в определенном направлении импульсом напряжения $V= \pm 6 \mathrm{~V}$ в течение $10 \mathrm{~s}$. На рис. 3 показаны вольтамперные характеристики PZT на подложках SOS и $\mathrm{Si}$ при положительной (кривые 1 и 4 ) и отрицательной 

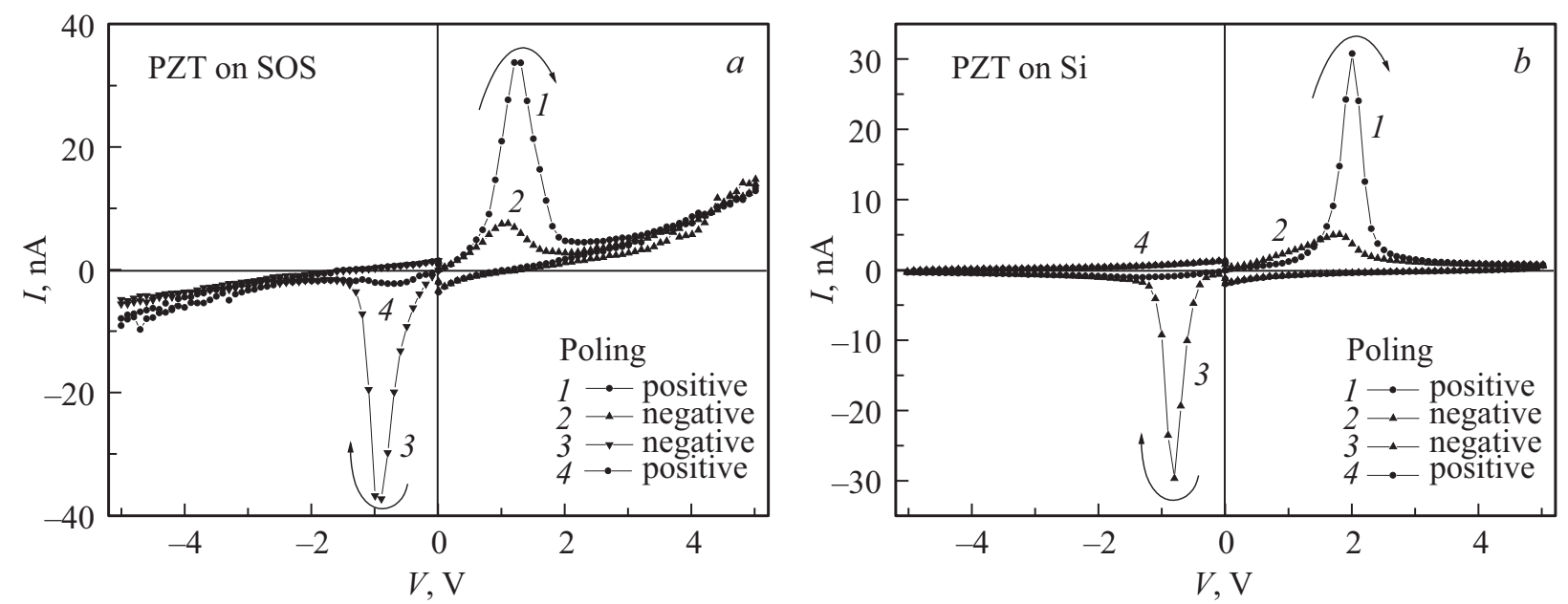

Рис. 3. Вольтамперные характеристики переходного тока, измеренные при разных предварительных поляризациях в пленке РZT на подложках $\operatorname{SOS}(a)$ и $\mathrm{Si}(b)$.

(кривые 2 и 3) предварительной поляризации. Для обоих направлений смещения наблюдается гистерезис тока по часовой стрелке, а также пики тока при совпадении направлений смещения и поляризации (кривые 1 и 3) на прямой ветви гистерезиса и отсутствие пиков на обратной ветви. Существование таких пиков в поликристаллических PZT-пленках со столбчатой структурой зерен свидетельствует о непроводящих границах зерен, как было показано ранее в [21]. Для объяснения появления указанных пиков нами была предложена диффузионнодрейфовая модель переноса носителей заряда в структурах $M / \mathrm{PZT} / M[22,23]$. В этой модели электроны, генерируемые кислородными вакансиями, захватываются глубокими центрами Ті при комнатной температуре и под действием электрического поля могут двигаться между атомами Ті посредством прыжкового механизма. Пик тока формируется, когда (a) обогащенный электронами слой пространственного заряда возникает у одного из контактов под действием поляризации и (b) в этот контакт втекает ток, т.е. он является катодом, что возможно только при совпадении направлений поляризации и смещения. Под действием приложенного внешнего напряжения электроны перераспределяются между слоями объемного заряда (CO3): концентрация электронов в обедненном слое увеличивается, а в обогащенном слое уменьшается, в результате чего обедненный СО3 становится тоньше и сопротивление пленки начинает падать. Так продолжается до тех пор, пока обогащенный слой у катода не превращается в обедненный, что вызывает увеличение сопротивления пленки и тем самым приводит к появлению пика тока. Поскольку для образования обогащенного СО3 требуются достаточно большие значения поляризации, то появление подобных пиков тока на кривых $I-V$ означает, что вектор по- ляризации сохраняет величину и направление по всей толщине пленки до электродов. Из рис. 3 видно, что обе пленки демонстрируют достаточно большие пики тока на кривых $I-V$, что свидетельствует об их высоких СЭ-характеристиках. Тем не менее, следует отметить, что пиковые значения тока у пленок PZT на SOS на $10-20 \%$ больше, чем соответствующие значения для PZT на $\mathrm{Si}$, что можно связать с меньшим значением переключаемой поляризации, что уже указывалось в предыдущем разделе.

\section{4. Измерение фотовольтаического тока}

Измерение фототока в условиях короткозамкнутой цепи - это известный способ изучения внутренних электрических полей в пленке. Измерялся зависящий от времени фототок короткозамкнутого конденсатора $M / \mathrm{PZT} / M$ при разных предварительных поляризациях. Вначале пленка деполяризовалась, затем поляризовалась в темноте импульсом смещения $V= \pm 6 \mathrm{~V}$ в течение $10 \mathrm{~s}$. После выключения смещения внешняя цепь оказывается закороченной. Когда переходный темновой ток уменьшался до нуля, полупрозрачный верхний электрод пленки облучался светом светодиодного источника с длиной волны $456.8 \mathrm{~nm}$, энергией фотона $2.7 \mathrm{eV}$ и мощностью накачки $4 \cdot 10^{16} \mathrm{~cm}^{-2} \mathrm{~s}^{-1}$, а протекающий при этом во внешней цепи фототок регистрировался с интервалом $1 \mathrm{~s}$. Результаты представлены на рис. 4. Видно, что в деполяризованном состоянии фототок в обеих пленках имеет отрицательное направление. Для PZT на SOS такое направление тока сохраняется при любом направлении поляризации пленки, в то время как для PZT на Si ток становится положительным при отрицательной поляризации. 

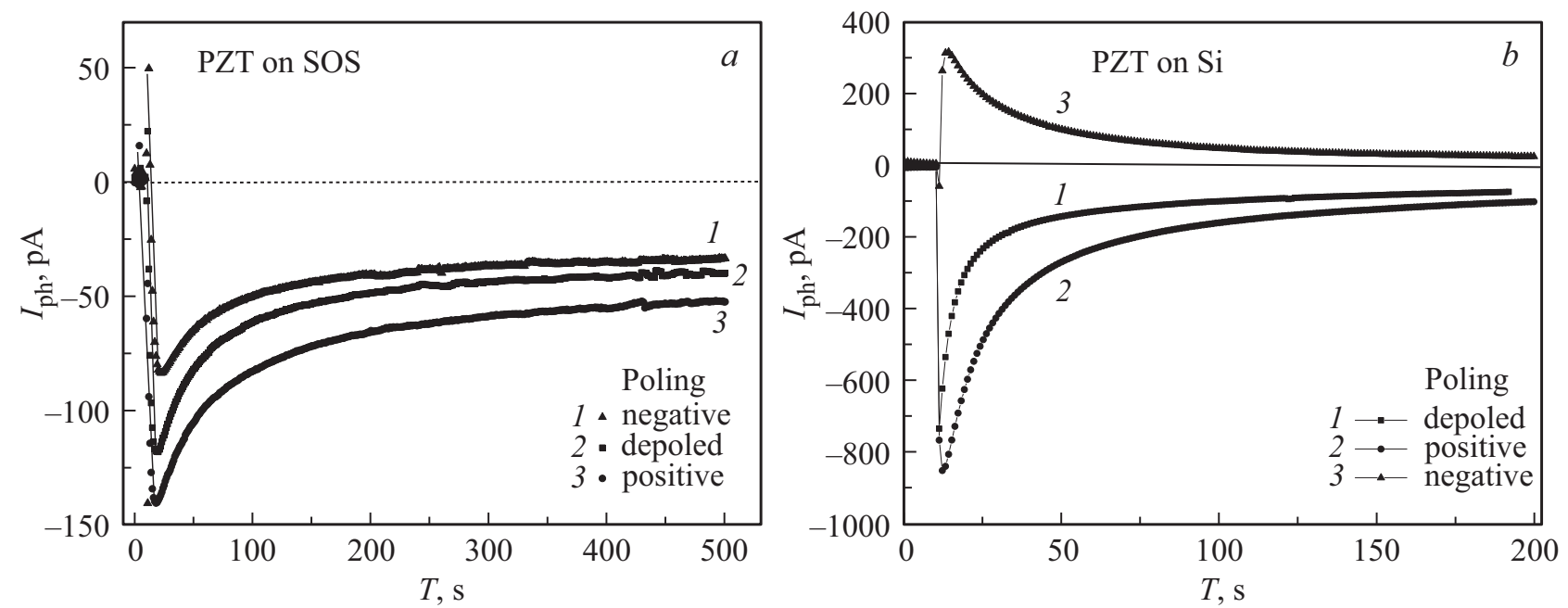

Рис. 4. Зависимости фототока от времени освещения пленок $\mathrm{PZT}$ на подложках $\operatorname{SOS}(a)$ и $\operatorname{Si}(b)$ при разных предварительных поляризациях.

Согласно объемному фотовольтаическому эффекту [24] фотовольтаический ток внутри СЭ-фазы должен течь в направлении поляризации. Однако это справедливо для объемных образцов, в тонких же пленках появляются дополнительные источники фотоэффекта, связанные с интерфейсом: возникающие механические напряжения, проявляющие себя в пьезоэффекте, и барьеры Шоттки на контактах $M / \mathrm{PZT}$ и PZT/M. Независимость направления фототока от направления поляризации позволяет считать, что именно эти факторы определяют фотоэффект в рассматриваемых пленках. Барьеры Шоттки создают слои объемного заряда, внутри которых существует электрическое поле, направленное в сторону электрода. Поскольку в настоящей работе рассматривались достаточно толстые пленки, то слои объемного заряда в них не пересекались и были разделены квазинейтральной областью, в которой положительно заряженные кислородные вакансии компенсировались отрицательным зарядом электронов, захваченных на уровни Ti $[22,23]$. Направление фототока определяется электрическим полем того СО3, чья толщина оказывается больше, а толщина $\mathrm{CO} 3$ зависит от величины контактной разности потенциала $\varphi_{C}$, концентрации вакансий, а также величины и знака поляризации. Отрицательный знак тока в деполяризованной пленке означает большую толщину СО3 у нижнего электрода, чем у верхнего, что, по-видимому, связано с большей величиной $\varphi_{C}$. Положительная поляризация увеличивает толщину СО3 у нижнего электрода, а отрицательная - уменьшает, что согласуется с вариациями фототока на рис. 4 при изменении направления поляризации. Образование максимума фототока связано с уменьшением толщины $\mathrm{CO} 3$ по мере накопления в них носителей заряда, образующихся в результате поглощения излучения. Заметим также, что в PZT на $\mathrm{Si}$ величина фототока оказывается больше, что можно объяснить большей величиной $\varphi_{C}$ у нижнего электрода вследствие большей величины деформации платины. Компенсируя фототок встречным током от внешнего источника, мы определили величину фото-ЭДС, которая лежит в диапазоне $120-160 \mathrm{mV}$ для разных пленок и которой соответствует некоторое эффективное среднее встроенное электрическое поле, величина которого составляет $\sim 10$ и $20 \mathrm{kV} / \mathrm{cm}$ для PZT на SOS и PZT на $\mathrm{Si}$ соответственно.

\section{5. Исследование деформаций и механических напряжений в пленках}

Чтобы получить представление о деформациях и механических напряжениях в пленках, были выполнены измерения изгиба подложек, а для PZT на Si были использованы также результаты измерений постоянных решетки методом рентгеновской дифракции.

3.5.1. Изгиб сапфировой подложки. Радиус изгиба сапфировой подложки измерялся на рентгеновском дифрактометре ДРОН-3 (излучение $\mathrm{Cu} K_{\alpha 1}$ ) путем регистрации смещения рефлекса (0006) от сапфира при последовательном линейном сканировании образца в рентгеновском пучке [25].

На рис. 5, $a$, показано полное угловое смещение $\Sigma(\delta \theta)$ рефлекса (0006), измеренное для каждой точки при сканировании вдоль образца. Изменение углового смещения является практически линейным, что свидетельствует о сферическом характере изгиба (измерения проводились при нескольких азимутальных положениях образца). По наклону кривой был определен радиус изгиба подложки $R=44.6 \mathrm{~m}$, откуда следует, что образец является выпуклым в сторону пленки РZT и поэтому биаксиальные напряжения, действующие в плоскости пленки, являются сжимающими. Поскольку толщина подложки $t_{\text {sub }}=510 \mu \mathrm{m}$ как минимум на два порядка 

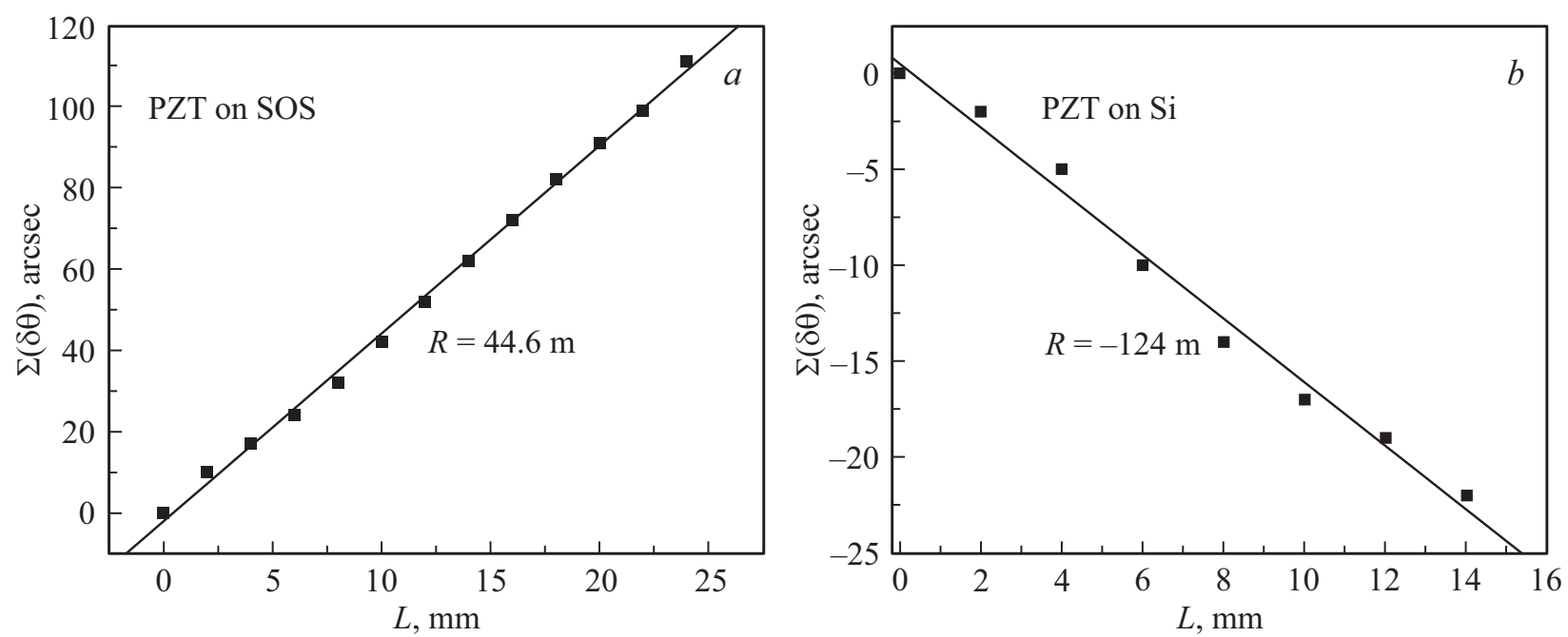

Рис. 5. Зависимость полного углового смещения $\Sigma(\delta \theta)$ пиков отражения (0006) от сапфира $(a)$ и $(004)$ от $\mathrm{Si}(b)$ при последовательном сканировании образца в рентгеновском пучке.

превосходит сумму толщин всех слоев, нанесенных на нее, $t_{\text {layer }}=1.21 \mu \mathrm{m}$, то для расчета остаточных напряжений в плоскости пленки можно использовать формулу Стоуни [26]:

$$
\sigma=-\frac{1}{6 R}\left(\frac{E}{1-v}\right)_{\text {sub }} \frac{t_{\text {sub }}^{2}}{t_{\text {layer }}}=-0.49 \mathrm{GPa},
$$

где $E$ и $v$ - модуль Юнга и коэффициент Пуассона для сапфира и $E /(1-v)=602 \mathrm{GPa}$. Полученное значение напряжения можно трактовать как среднее по слою $\mathrm{Si} / \mathrm{SiO}_{2} / \mathrm{Pt} / \mathrm{PZT}$. Применительно к Pt это означает, что изгиб подложки вызывает в Pt напряжения сжатия в еe плоскости. С другой стороны, РZT стремится ее растянуть из-за несоответствия параметров решеток. Повидимому, этим можно объяснить достаточно высокое совершенство Pt в случае сапфировой подложки, отмеченное в измерениях методом рентгеновской дифракции, что, в свою очередь, способствует улучшению свойств пленки PZT.

В соответствии с прямым пьезоэффектом, напряжения сжатия в плоскости пленки приводят к появлению заряда в перпендикулярном направлении. В рассматриваемом случае этот заряд равен $d_{31} \sigma \approx 4 \mu \mathrm{C} / \mathrm{cm}^{2}$, где $d_{31}=-82 \cdot 10^{-12} \mathrm{C} / \mathrm{N}$ - пьезомодуль для золь-гельпленок PZT [27]. Полученная величина существенно больше непереключаемой поляризации, но близка к абсолютному значению остаточной поляризации $P_{\text {dep }}$ в деполяризованном состоянии пленки (рис. 2, $a$ ). Однако сделать из этого какие-то определенные выводы о связи $P_{n o n-s w}$ и $P_{\text {dep }}$ с изгибом подложки не представляется возможным.

3.5.2. Изгиб кремниевой подложки. Изгиб кремниевой подложки также измерялся путем регистрации полного смещения дифракционного пика $\mathrm{Si}$ (004) при последовательном линейном сканировании образца в пучке рентгеновских лучей, рис. 5, $b$. Образец вогнут со стороны пленки PZT с радиусом изгиба $R=-124$ m, и поэтому биаксиальные напряжения, действующие в плоскости пленки, являются растягивающими. Используя опять для оценки напряжения формулу Стоуни и учитывая, что в этом случае $t_{s u b}=690 \mu \mathrm{m}$, $t_{\text {layer }}=0.67 \mu \mathrm{m}$ и $E /(1-v)=180.5 \mathrm{GPa}[28]$, получаем $\sigma=0.172 \mathrm{GPa}$. Таким образом, в противоположность PZT на SOS, PZT на Si оказывается растянутой в плоскости $\mathrm{X}_{1} 0 \mathrm{X}_{2}$ (в связанной с подложкой декартовой системе координат $\mathrm{X}_{1} \mathrm{X}_{2} \mathrm{X}_{3}$, где ось $\mathrm{X}_{3}$ направлена вдоль объемной диагонали (111) тетрагональной структуры PZT) и сжатой в направлении нормали $0 \mathrm{X}_{3}$, т. е. вдоль оси (111). Для оценки деформаций в пленке можно воспользоваться формулами $\varepsilon_{11}^{\text {bend }}=\varepsilon_{22}^{\text {bend }}=\left(s_{11}+s_{12}\right) \sigma$ и $\sigma_{33}^{\text {bend }}=-(2 v / E)_{\mathrm{PZT}} \sigma$, где $s_{11}$ и $s_{12}-$ упругие податливости РZT, $E$ - модуль Юнга PZT, v - коэффициент Пуассона PZT [29]. В результате получаем $\varepsilon_{33}^{\text {bend }}=-0.00152$, a $\varepsilon_{11}^{\text {bend }}=\varepsilon_{22}^{\text {bend }}=0.00117$.

3.5.3. Оценка деформаций в PZT на Si методом XRD. Как указывалось выше, параметры решетки PZT, найденные из результатов XRD-измерений, отличаются от табличных значений, что позволяет получить оценки трех компонент тензора деформаций в кристаллофизической системе координат $\overline{\mathrm{X}}_{1} \overline{\mathrm{X}}_{2} \overline{\mathrm{X}}_{3}$, где оси $\overline{\mathrm{X}}_{1}$ и $\overline{\mathrm{X}}_{2}$ соответствуют направлениям (100) и $(010)$, а ось $\overline{\mathrm{X}}_{3}$ - направлению $(001): \bar{\varepsilon}_{11}^{\mathrm{XRD}}=\bar{\varepsilon}_{22}^{\mathrm{XRD}}=0.0042236$ и $\bar{\varepsilon}_{33}=-0.00869$. Чтобы сравнить эти деформации со значениями, полученными из измерения изгиба подложки, необходимо перейти из системы координат $\overline{\mathrm{X}}_{1} \overline{\mathrm{X}}_{2} \overline{\mathrm{X}}_{3}$ в систему координат $\mathrm{X}_{1} \mathrm{X}_{2} \mathrm{X}_{3}$, и найти указанные компоненты тензора в этой системе координат. Они определяются по формулам $\varepsilon_{i j}^{\mathrm{XRD}}=\alpha_{i m} \alpha_{j n} \bar{\varepsilon}_{m n}^{\mathrm{XRD}}$, где $\alpha_{i j}-$ направляющие косинусы, а по повторяющимся индексам производится суммирование. Однако чтобы это сделать, необходимо знать деформации сдвига $\bar{\varepsilon}_{i j, i \neq j}^{\mathrm{XRD}}$ в системе координат $\overline{\mathrm{X}}_{1} \overline{\mathrm{X}}_{2} \overline{\mathrm{X}}_{3}$. Для решения 
этой проблемы положим, что деформации сдвига $\varepsilon_{i j, i \neq j}^{\mathrm{XRD}}$ в системе координат $\mathrm{X}_{1} \mathrm{X}_{2} \mathrm{X}_{3}$ равны нулю. Подобное допущение является вполне оправданным, поскольку толщина пленки существенно меньше ее продольных размеров. Более того, оно используется практически во всех работах, рассматривающих деформации в тонких пленках. Учитывая симметричность тензора деформации, из трех уравнений $\alpha_{i m} \alpha_{j n} \bar{\varepsilon}_{m n}=0, i \neq j, i=1,2,3$ получим $\bar{\varepsilon}_{12}^{\mathrm{XRD}}=0$ и $\bar{\varepsilon}_{13}^{\mathrm{XRD}}=\bar{\varepsilon}_{23}^{\mathrm{XRD}}=-0.01256$. Отсюда следует, что в деформированной элементарной ячейке угол между ребрами $a$ остался равным 90 градусов, а угол между ребрами $a$ и $c$ увеличился на 0.7 градуса. Что же касается деформаций $\varepsilon_{11}^{\mathrm{XRD}}, \varepsilon_{22}^{\mathrm{XRD}}, \varepsilon_{33}^{\mathrm{XRD}}$, то их значения оказываются равными соответствующим значениям деформаций в кристаллофизической системе координат. Таким образом, оба подхода дают качественно одинаковые результаты: пленка сжата в направлении нормали и растянута в своей плоскости. Однако величины $\varepsilon_{i i}^{\mathrm{XRD}}$ значительно больше $\varepsilon_{i i}^{\text {bend }}$. Чем вызывается такое различие, остается неясным. Можно предположить, что разность $\varepsilon_{33}^{\mathrm{XRD}}-\varepsilon_{33}^{\text {bend }}=-0.00717$ определяет деформацию $\varepsilon_{33}$, связанную с напряжением $\sigma_{33}$, действующим поперек пленки. Можно считать, что на поверхности пленки $\sigma_{33}=0$, поскольку верхний электрод является очень тонким. Следовательно, $\varepsilon_{33}$ также равна нулю на поверхности. А учитывая, что значения деформаций $\varepsilon_{i i}^{\mathrm{XRD}}$ и $\varepsilon_{i i}^{b e n d}$ являются средними по толщине пленки, мы можем найти градиент деформации $d \varepsilon_{33} / d x_{3}=-2\left(\varepsilon_{33}^{\mathrm{XRD}}-\varepsilon_{33}^{\text {bend }}\right) / h=683 \mathrm{~cm}^{-1}$, где $h$ - толщина пленки. Поскольку знак градиента положительный, как и знак непереключаемой поляризации $P_{n o n-s w}$, то имеются все основания полагать, что возникновение $P_{n o n-s w}$ связано с флексоэлектрическим эффектом. Как следствие этого, мы можем получить оценку флексоэлектрического коэффициента $\mu_{11}=P_{n o n-s w}\left(d \varepsilon_{33} / d x_{3}\right)^{-1}=0.0154 \mu \mathrm{C} / \mathrm{cm}$, что близко к величине флексоэлектрического коэффициента для PZTкерамики $\mu_{12}=0.014 \mu \mathrm{C} / \mathrm{cm}[30]$.

\section{4. Заключение}

Показано, что лучшее качество тонких PZT-пленок достигается на сапфировой подложке: она обеспечивает практически ненапряженный слой $\mathrm{Pt}$, на котором формируется текстура (111) зерен перовскита, а также симметричные петли гистерезиса с высокой остаточной поляризацией. Пленки PZT на $\mathrm{Si}$ хотя и демонстрируют преимущественную текстуру (111), однако, показывают меньшие значения остаточной поляризации и проявляют асимметрию петель гистерезиса, вызванную наличием положительной непереключаемой поляризации. Измерения фототока и фото-ЭДС показывают, что фотоэффект связан с электрическими полями, возникающими в слоях объемного заряда около электродов. Выпуклый изгиб сапфировой подложки производит сжатие пленки в ее плоскости, что вызывает небольшой пьезоэффект, слабо влияющий на поляризационные свойства пленок. В случае подложки $\mathrm{Si}$ оценки деформаций, полученные из XRD-измерений и измерений изгиба подложки, вопервых, показывают, что пленка сжата в направлении нормали и растянута в своей плоскости, а во-вторых, дают возможность определить деформацию, связанную с напряжением, действующим поперек пленки. Получена оценка градиента деформации вдоль оси (111), которая позволила связать асимметрию петель гистерезиса с флексоэлектрической поляризацией и найти величину флексоэлектрического коэффициента $\mu_{11}$ для золь-гель пленок PZT, которая оказалась равной $0.0154 \mu \mathrm{C} / \mathrm{cm}$ и весьма близкой к значению коэффициента $\mu_{12}$ для PZT-керамики.

\section{Финансирование работы}

Авторы Л.А. Делимова, Н.В. Зайцева и В.С. Юферев благодарят за поддержку РФФИ, проект № 19-02-00148, Д.С. Серегин, К.А. Воротилов и А.С. Сигов благодарят РФФИ, проект № 19-29-03058.

\section{Конфликт интересов}

Авторы заявляют об отсутствии конфликта интересов.

\section{Список литературы}

[1] Д.А. Абдуллаев, Р.А. Милованов, Р.Л. Волков, Н.И. Боргардт, А.Н. Ланцев, К.А. Воротилов, А.С. Сигов. Рос. техн. журн. 8, 5, 44 (2020).

https://doi.org/10.32362/2500-316X-2020-8-5-44-67

[2] N. Setter, D. Damjanovic, L. Eng, G. Fox, S. Gevorgian, S. Hong, A. Kingon, H. Kohlstedt, N.Y. Park, G.B. Stephenson, I. Stolitchnov, A.K. Taganstev, D.V. Taylor, T. Yamada, S. Streiffer. J. Appl. Phys. 100, 5, 051606 (2006). https://doi.org/10.1063/1.2336999

[3] G.L. Smith, J.S. Pulskamp, L.M. Sanchez, D.M. Potrepka, R.M. Proie, T.G. Ivanov, R.Q. Rudy, W.D. Nothwang, S.S. Bedair, Ch.D. Meyer, R.G. Polcawich. J. Am. Ceram. Soc. 95, 6, 1777 (2012). doi: 10.1111/j.1551-2916.2012. 05155.x

[4] I. Kanno. Jpn. J. Appl. Phys. 57, 4, 040101 (2018). doi: 10.7567/JJAP.57.040101

[5] R.R. Benoit, R.Q. Rudy, J.S. Pulskamp, R.G. Polcawich, S.S. Bedair. J. Micromech. Microeng. 27, 8, 083002 (2017). doi: 10.1088/1361-6439/aa710b

[6] Chemical Solution Deposition of Functional Oxide Thin Films / Eds T. Schneller, R. Waser, M. Kosec, D. Payne. Springer (2013). doi: 10.1007/978-3-211-99311-8

[7] B.-Y. Nguyen, G. Celler, C. Mazuré. J. Integr. Circuits Syst. 4, 2, 51 (2009). DOI: 10.29292/jics.v4i2.297

[8] G.K. Celler. ECS Trans. 19, 4, 3 (2009). sci-hub.do/10.1149/1.3117387

[9] https://www.soitec.com/en/

[10] T. Nakamura, H. Matsuhashi, Y. Nagatomo. Oki Tech. Rev. 71, 4, 66 (2004).

[11] George Imthurn. The History of Silicon-on-Sapphire. $\mathrm{http} / / / \mathrm{www}$. admiral-microwaves.co.uk/pdf/peregrine/ History_SOS_73-0020-02.pdf 
[12] H.W. Jiang, P. Kirby, Q. Zhang. Fabrication of PZT actuated cantilevers on silicon-on-insulator wafers for a RF microswitch. Micromachining and Microfabrication Process Technology VIII. San Jose, California, USA (2003). P. 165. https://dspace.lib.cranfield.ac.uk/bitstream/handle/1826/1485/ Fab\%20PZT-silicon-on-insular\%20wafers-2003.pdf?sequence $=3 \&$ isAllowed $=\mathrm{y}$

[13] Y. Hishinuma, E.-H. Yang. J. Microelectromech. Syst. 15, 2, 370 (2006). DOI: 10.1109/JMEMS.2006.872229

[14] G.J.T. Leighton, P.B. Kirby, C.H.J. Fox. Appl. Phys. Lett. 91, 18, 183510-1/183510-3 (2007).

[15] T. Bland, J. Obhi, A. Patel, P. Kirby, M. Robinson, J. Kerr. Microelectron. Eng. 29, 1-4, 29 (1995).

[16] R.R. Benoit, C.Y. Cheng, R.Q. Rudy, R.G. Polcawich, J.S. Pulskamp, D.M. Potrepka, B.M. Hanrahan, S. TrolierMcKinstry. Sputtered Lead Zirconate Titanate Thin Films Deposited on Silicon-on-Sapphire Substrates. IEEE MTTS International Microwave Workshop Series on Advanced Materials and Processes for $\mathrm{RF}$ and $\mathrm{THz}$ Applications (IMWS-AMP 2018). (July 16-18, 2018). Ann Arbor, MI, USA.

[17] M. Adachi, T. Matsuzaki, T. Yamada, T. Shiosaki, A. Kawabata. Jpn. J. Appl. Phys. 26, 4, 550 (1987). doi: 10.1143/JJAP.26.550

[18] W. Braun, B.S. Kwak, A. Erbil, J.D. Budai, B.J. Wilkens. Appl. Phys. Lett. 63, 4, 467 (1993). doi: 10.1063/1.110026

[19] G. Han, J. Ryu, W.-H. Yoon, J.-J. Choi, B.-D. Hahn, J.-W. Kim, D.-S. Park, C.-W. Ahn, S. Priya, D.-Y. Jeong. J. Appl. Phys. 110, 124101 (2011). doi: 10.1063/1.3669384

[20] N.M. Kotova, K.A. Vorotilov, D.S. Seregin, A.S. Sigov. Inorg. Mater. 50, 6, 612 (2014).

[21] L.A. Delimova, E.V. Guschina, D.S. Seregin, K.A. Vorotilov, A.S. Sigov. J. Appl. Phys. 121, 224104 (2017). http://dx.doi.org/10.1063/1.4985177

[22] L.A. Delimova, V.S. Yuferev. J. Appl. Phys. 124, 18, 184102, (2018). https://doi.org/10.1063/1.5052613

[23] L.A. Delimova, V.S. Yuferev. J. Phys. Conf. Ser. 1400, 055003 (2019). doi: 10.1088/1742-6596/1400/5/055003

[24] В.М. Фридкин, Б.Н. Попов. УФН 126, 657 (1978).

[25] G.A. Rozgonyi, T. Ciesielka. Rev. Sci. Instrum. 44, 1053 (1973).

[26] G.G. Stoney. Proc. R. Soc. Lond. A 82, 172 (1909).

[27] X. Jiang, W. Huang, Sh. Zhang. Nano Energy 2, 1079 (2013). http://dx.doi.org/10.1016/j.nanoen.2013.09.001

[28] W.A. Brantley. J. Appl. Phys. 44, 1, 534 (1973).

[29] Б. Яффе, У. Кук, Г. Яффе. Пьезоэлектрическая керамика / Под ред. Л.А. Шувалова. Мир, М. (1974). С. 136.

[30] W. Ma, L.E. Gross. Appl. Phys. Lett. 86, 072905 (2005).

Редактор Е.Ю. Флегонтова 